\title{
Some Features of China's Small Hydropower
}

\author{
Tong Jiandong
}

Abstract: China developed small hydropower (SHP) with its own resources instead of importing advanced technology from other countries. SHP has been instrumental in rural electrification. In 1997, SHP accounted for $28.6 \%$ of whole exploitable hydro energy in China. The peculiarities of SHP of China are:

1. Decentralized approach for SHP development with county as basic unit,

2. Special policies and strategies adopted

- self construction, self management and self consumption

- rural hydropower development fund set up - levy of 2 cents of each kWh

- electricity generates electricity policy

3. Various Channels of investment are mobilized

To exploit China's experience in SHP the International Network on Small Hydropower (IN-SHP) was established in Hangzhou, China.

Key words: Small hydropower, rural electrification, China

$\mathbf{S}$ mall Hydropower (SHP) development in China started as early as 1950 in conjunction with the agricultural and rural development progress in China. Electricity demand in rural China was very low at that time. There was more time for the Chinese people to prepare our own knowledge in planning, implementation \& operation at low cost level for SHP than to introduce advanced technology from other countries, and our peculiarities in SHP were formed. The specific features of SHP development in China may be briefed as follows:

\section{Decentralized approach for SHP development with county as the basic unit}

Compared with the management system of other developing countries, the system approach of SHP development in China is a decentralized one - mainly on local level. Except the main policy and strategy, as well as national overall planning which have always been stipulated by the central government, all other aspects such as project planning, project development, implementation and management, operation \& maintenance are decentralized to different government levels; i.e., province, prefecture, county or even township . The approach is a strategy developed on the basis of 'local-based, SHP-first, and service-oriented' policy. Its main idea is 'walking with two legs'-the SHP exploitation mainly depending on the local resources in the spirit of selfreliance on the one hand, and the extension of large grid on the other hand. The approach has effectively promoted the decentralized administrative structures of electricity supply in China rural area, with the county as basic unit and having electricity supplied by the national grid, the local grid and isolated SHP stations.

\section{Special policies and strategies adopted}

The Chinese government has consistently attached great importanceto stipulating and regulating a series of supporting policies for the development of SHP since early 1950s. The Policy of 'Self-construction, self-management, and self- consumption' has been well-known for SHP development since early 1960s. 'Self-construction' means that local authorities/entities or local people (collective or private) are encouraged to plan and construct SHP stations utilizing the local resources, technologies and raw materials, particularly local fund. In some places, even the SHP equipment in need are produced by local entities themselves. 'Self-management' defines that: either local authorities/ entities or local people (collective or private) who invest and construct the SHP stations owns the stations. 'Self consumption' implies that there should be a SHP market; i.e., SHP stations should have their own distribution/ supply area (concession area) instead of selling all the energy to the national grid for distribution. Below are the main policies:

- Efforts by leaders. Each county sets up leading group led by the county administrator for Rural electrification (RE), to make important decisions for RE, construction, fund and manpower mobilization.

- Rural hydropower development funds set up. In 1991, the Chinese Ministry of Water issued a document to levy 2 cent for each kWh generated in the rural hydropower base for rural hydropower development funds.

- 'Electricity generates electricity' policy. All the profits from SHP are sent to the enterprise themselves for reproduction. This policy has been in effect for 20 years, playing an important role in developing rural SHP.

- Tax policy. New tax methods were introduced in 1994. Before that period, only $5 \%$ as business tax was levied from SHP station. Starting from 1994, it has been changed into $6 \%$ as the value added tax, much favorable than the $17 \%$ tax for the large hydropower stations or grids. The income tax is $33 \%$ but some provinces levy only half of it.

- Pricing policy. The energy generated from SHP is not within the planned amount and may participate in market adjustment. The price may be determined by the cost, tax 
and some profits.

- Loan policy. The Agricultural Bank of China lists special funds as loans for developing rural SHP

- Protection policy of areas supplied by SHP. The state decides that large grids supports SHP which are connected with the large grids in some areas to supplement each other.

\section{Various channels of capital investment}

SHP development needs large amount of money, and various channels are needed to this end:

- Multi-channel, multi-level and multi-pattern for fund collection. The individual farmers, groups and enterprises and funds outside the county are encouraged to invest, adopting the policy of 'those who invest own and benefit'.

- Applying share-holding corporation system for fund mobilization. Starting about 6 years ago, about 80 share holding corporation for developing SHP stations or grids have been set up. There are over 300 share-holding cooperative \& enterprises patterns for SHP construction. These new patterns are effective in attracting funds.

- Support from the government. Governments from the central, provincial to the county level invest hundreds of million RMB in SHP construction. These loans with low interest rate are returned within 10 years or so.

- Rolling investment by the enterprises. Nearly each county has some SHP stations and grids, and each year profits are gained. These profits can be used as inputs. Such fund reached 482 million yuan in 1996.

- Funds mobilized by the farmers themselves. A solution to the shortage of construction fund is to mobilize fund by local farmers themselves. Generally speaking, the funds for the transmission line system of less than $10 \mathrm{kV}$ is mobilized by the farmers. In the very poor hilly areas, governments provide some supports. It is a popular pattern for farmers to participate in the fund mobilization and engineering construction. Some counties have made it a rule that each farmer who has normal labor ability is obliged to work for public and water conservancy facilities for no less than 8-10 working days per year. In some other counties, a farmer can become a shareholder with his (her) volunteer work for construction of SHP stations and power grid.

- Loan from the financial agencies. This is the main fund body formed within the recent 10 years. Agricultural and construction banks set special funds for SHP construction.

In 1991, the sources of investment of multi-channels and multi-levels are such: the subsidy from central government only accounted for $5.7 \%$, local mobilization was about $30 \%$, while bank loans was as high as $45 \%$. Entering into the 1990s, with the further development of market economy, the financing function of the financial institutions will become more and more remarkable and SHP will be developed and operated in a more commercialized way.

\section{Close relation to Chinese $\mathrm{RE}$}

At the beginning of 1970s, when food problems were mitigated, the local/rural industries started to boom. Demands for energy, electricity in particular, increased with each passing day in rural areas. Rural electrification has become a significant issue for the economic development in Rural China.

However, it will be unrealistic as well as uneconomic to depend wholly upon the central government or wait for the extension of large grid to realize rural electrification. Therefore, by pursuing the policy of 'walking with two legs', it will be conducive to the state, the collective and the individual to let those hilly areas having rich SHP resources become electrified first, and to improve the consumption level in those areas. In 1982, the central government decided to launch pilot projects in over 100 counties of rich SHP sources. It is from then that the main orientation of realizing rural electrification through SHP exploitation became clear.

This Chinese mode of RE with the characteristic of SHP exploitation mainly depending on the local resources has three basic features.

i. Power sources mainly relying on the rich SHP resources in China;

ii. Construction mainly depending upon the local masses, in pursuit of the 'three self' principle; i.e., 'self-construction, self-management, and self-consumption'. In this way, the initiative of local people in SHP exploitation is protected.

iii. The power consumption level must fit the living standard of farmers.

The practice of realizing rural electrification through SHP exploitation envisages the prospect of China rural development, though the electrification in China is still low compared with the level of developed countries, and it is no easy job to realize electrification for the whole 900 mil. rural population.

\section{Cost-effective SHP technology}

The swift development and salient benefits of Chinese RE result from application of new technology, new products, and a series of practical indigenous SHP technology is formed in conformity with the contemporary rural development level. They are:

- The formation of primary RE standards. Annual energy consumption per capita and per household exceeds 200 kWh respectively; Electricity coverage over 90\%, energy reliability over $85 \%$. The scientific value of the standard is the direct connection between RE and economic growth and increase of dwellers' living standard; It reflects the simultaneous development of generation, supply and consumption of power.

- A wholly new method in RE planning has been discovered. 
As RE in China is mainly surplus power output in wet season and the inadequacy in dry season. After study, 'doubleway adjustment' is adopted; i.e., selecting the optimized grid operation as the target instead of the benefit of an individual SHP station, to meet the growing demand. In site selection, both the potential power and energy are considered for the balance of it. So in quite a few of the counties, iron-alloy, silicon-iron plants which can be adjusted to the use of energy to a degree are arranged between the flood and dry season. If it is still short to adjust the balance between power and energy, SHP stations with regulating capacity; i.e., a reservoir, or thermal power stations should be considered. In places where large grids are available, the power can be sent to the large grids in flood season while in dry season the large grids can supplement the power.

- The stipulation of the technical standards, norms, rules and criteria in each process of work from initial stage to construction, installation, acceptance and operation. These technical rules and standards guarantee the quality, effectiveness and the completion of SHP construction on time.

- Dissemination of new technology. These include: rolling concrete arch dam, plate rock filling dam, pitch-core rockfilling dam, unmanned SHP stations and substations, F-plastics tilts, automatic trash rack, grid dispatching automation, optimum operation dispatching, transbasin hydropower exploitation, water complex optimized operation, cascade SH exploitation, computerized feecollecting etc. In electro-mechanic products, efficient runners, simplified turbine operators, brushless excitation systems, sulfur hexafluoride switches, etc. are developed. The application of these products not only saves money but also shortens the construction period and increases the power reliability.

- Talents are cultivated quickly. Twelve institutions of higher learning are specially oriented for trainees from counties for RE. After graduation they are expected to be back to their hometown for RE construction. Meanwhile some working staff are selected for further training with a duration of 2 years. Electricians are also sent to county technical schools for training in turn. Presently, those RE counties are capable of designing, constructing, installing SHP stations with capacity less than $2 \mathrm{MW}$ and a transmission system of $35 \mathrm{kV}$. Some $\mathrm{RE}$ counties are capable of designing, constructing, installing SHP stations with a capacity less than $30 \mathrm{MW}$ and transmission system of $110 \mathrm{kV}$. Some even can go into overseas contract of engineering.

\section{Formation of local grids}

In China, attention is paid to develop the supply area of SHP, and county grids or prefecture grids has been formed. A county grid ranges from 20 to $50 \mathrm{MW}$ in general. Presently about $64.4 \%$ of the SHP installed capacity and $66.9 \%$ of SHP generation have been integrated into the county or prefecture grids. Three channels of electric power supply/ distribution has formed:

- For counties mainly supplied by SHP. Unified generation, supply and distribution system is adopted and managed by county level entity, either Bureau of Water Resource (BWR) and/or SHP Corporation. They have a local grid with their own distribution system (usually 35/10 - $0.4 \mathrm{kV}$ ) and supply areas. They are able to set up an independent tariff system for their own. These local grids, if possible, are usually connected with national grid at one point for exchange of energy. Whole country still depend on national grid for electricity supply, which accounts for $85 \%$ of the total power supply in rural China.

- For counties supplied by the national grid through bulk -selling to a local grid. In regions where there are not many medium and small hydropower stations, and electric power is in short, the electricity to rural areas is supplied by the national grid through bulk-selling to a local grid.

In about 800 counties where the electric power is mainly supplied by SHP, $80 \%$ of the electric power is supplied by county grids or prefecture grids.

\section{The prospect of SHP}

The SHP construction in China has developed from utilizing the rich SHP resources in hilly areas to solve the electricity shortage there in conjunction with the construction of small sized water conservancy projects in these areas to such a large scale construction as thousands of MW installed each year for continuous ten years, and has accelerated the social and economic development in local areas.

Table 1 is the SHP development data of various provinces of China in 1997. It can seen that in 1997 SHP has accounted for $28.6 \%$ of the whole exploitable energy in China, much more than that of large and medium sized hydropower. The least developed province in terms of SHP is Tibet, about $1 \%$, while the provinces where SHP is most developed and the SHP resource is richest are Guangdong, Fujian, Sichuan and Hunan Provinces and Guangxi Autonomous Region.

In 1996, the exploitable potential only include the site with installed capacity up to $25 \mathrm{MW}$, whereas the developed stations in the table also contain some stations between $25 \mathrm{MW} \& 50 \mathrm{MW}$ with total installed capacity of 1,661.9 MW, so the real ratio for developed SHP is only about $24.3 \%$.

The SHP development also enables the continuous growth of local grids in China, which results in the great change in composition of rural consumers in China. It guarantees the rapid and sustainable increase of demands for electricity in the county-run enterprises, township enterprises and rural households. It shows SHP has been playing a more and more important role in the scio-economic development in rural China and environmental protection. SHP is no longer a mere technology or method to solve the rural energy problem, but a new industry playing significant role in social, economic, and environmental development, and a mainstay industry for realizing material and cultural civilizations in 


\begin{tabular}{|c|c|c|c|c|}
\hline Region & Exploitable & Developed & Ratio (\%)* & $\begin{array}{c}\text { Annual Output } \\
\text { (GWh) }\end{array}$ \\
\hline Whole Nation & 71,870 & 2052.0 & 28.6 & $68,340.4$ \\
\hline Beijing & 90 & 56.7 & 63.0 & 67.9 \\
\hline Hebei & 939.3 & 253.6 & 27.0 & 467.7 \\
\hline Shanxi & 581 & 138.7 & 23.9 & 245.5 \\
\hline Inner Mongolia & 387 & 42.1 & 10.9 & 89.2 \\
\hline Liaoning & 429 & 175.4 & 40.9 & 434.0 \\
\hline Jilin & 1,888 & 179.6 & 9.5 & 454.7 \\
\hline Heilongjiang & 728 & 127.2 & 17.5 & 376.7 \\
\hline Jiangsu & 112 & 32.7 & 29.2 & 52.9 \\
\hline Zhejiang & 3,227 & 1226.8 & 38.0 & 2697.7 \\
\hline Anhui & 685 & 225.9 & 33.0 & 371.1 \\
\hline Fujian & 3,594 & 2249.6 & 62.6 & 8040.9 \\
\hline Jiangxi & 3,083 & 974.4 & 31.6 & 2757.2 \\
\hline Shandong & 215 & 77.4 & 36.0 & 44.9 \\
\hline Henan & 1,031 & 277.7 & 26.9 & 492.7 \\
\hline Hubei & 4,036 & 1226.9 & 30.4 & 3188.1 \\
\hline Hunan & 4,146 & 1809.8 & 43.7 & 6631.4 \\
\hline Guangdong & 4,166 & 2694.4 & 64.7 & 9389.2 \\
\hline Guangxi & 2,322 & 1226.4 & 52.8 & 4972.5 \\
\hline Guizhou & 2,554 & 770.7 & 30.2 & 2662.3 \\
\hline Yunnan & 10,250 & 1682.8 & 16.4 & 6557.0 \\
\hline Tibet & 16,000 & 162.3 & 1.0 & 385.5 \\
\hline Shaanxi & 1,569 & 279.9 & 17.8 & 581.4 \\
\hline Gansu & 1,089 & 274.9 & 25.2 & 851.9 \\
\hline Qinghai & 2,000 & 157.2 & 7.9 & 499.0 \\
\hline Ningxia & 23 & 6.0 & 26.1 & 10.8 \\
\hline Xinjiang & 3,979 & 570.8 & 14.3 & 1826.1 \\
\hline Hainan & 397 & 195.8 & 49.3 & 644.2 \\
\hline Tianjing & ----- & 5.8 & ----- & 20.8 \\
\hline
\end{tabular}

Table 1 SHP Development in 1997 (Unit: MW)

China’s rural area.

At the initiative of UNDP, UNIDO and the Ministry of Water Resource and the Ministry of Foreign Trade and Economic Cooperation of China, the International Network on Small Hydropower (IN-SHP) was established in Hangzhou, with its headquarters seated in Hangzhou International Center on Small Hydropower (HIC). IN-SHP consists of six Focal Points in five continents, namely, ESHA, OLADE, CANMET, SOPAC, AFPREN, and HRC. In the meantime, under the six Focal Points, over 60 country centers have been developed, in charge of the liaison, information exchange, policy coordination, technology demonstration, training, and economic and trade cooperation worldwide. Since its establishment, IN-SHP has created more and more opportunities for international cooperation in the area of small hydropower.

Tong Jiandong is Director General of IN-SHP and International Centre on Small Hydropower, Hanghzou, China. He is internationally renowned authority in small hydropower development. He has written some Books including Small Hydropower: China's Practice.

Corresponding address: tongjiandong@gmail.com

\section{Notes}

1. This article was previously published on the Internet at www.lanl.gov/chinawater/documents/tongjiandong.pdf. 